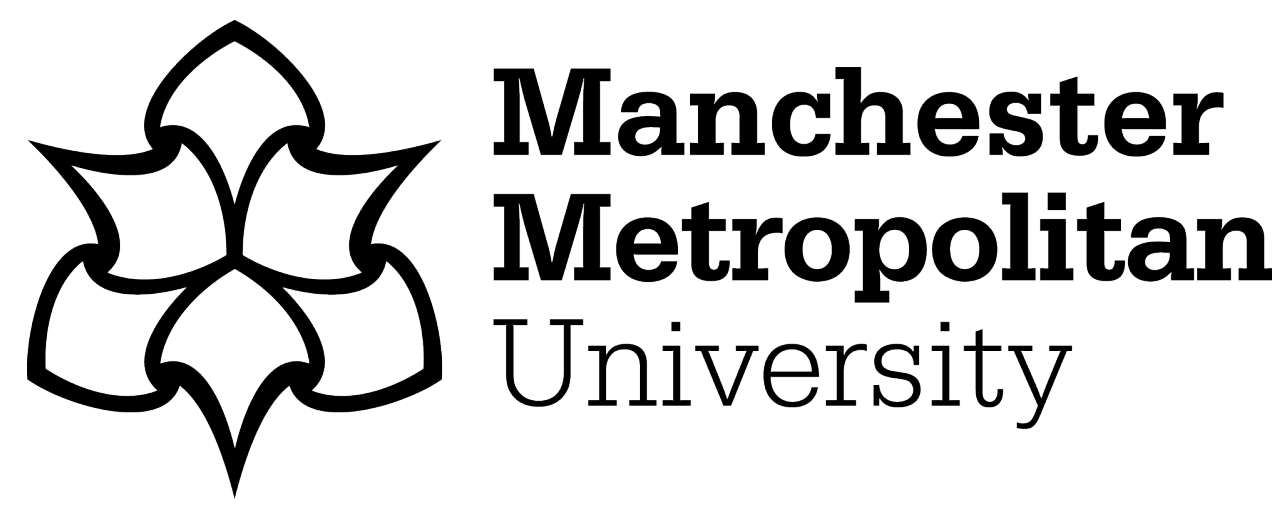

Rybakov, V (2014) Multi-agent non-linear temporal logic with embodied agent describing uncertainty. In: 8th International Conference KES-AMSTA 2014, 18 June 2014 - 20 June 2014, Chania, Greece.

Downloaded from: https://e-space.mmu.ac.uk/600862/

Version: Accepted Version

Publisher: Springer

DOI: https://doi.org/10.1007/978-3-319-07650-8_10

Please cite the published version 


\title{
Multi-Agent Non-Linear Temporal Logic with Embodied Agent describing Uncertainty
}

\author{
Vladimir Rybakov ${ }^{a, b}$ \\ (a) School of Computing, Mathematics and DT, Manchester Metropolitan University, \\ John Dalton Building, Chester Street, Manchester M1 5GD, U.K. and (b - part time) \\ Siberian Federal University, 79 Svobodny Prospect, Krasnoyarsk, 660041, Russia. \\ E-mail: V.Rybakov@mmu.ac.uk
}

\begin{abstract}
We study non-linear temporal multi-agent logic $\mathbf{T}_{\mathbf{K n}}^{\mathbf{E m}, \mathbf{I n t}}$ with embodied agent. Our approach models interaction of the agents and various aspects for computation of uncertainty in multi-agent environment. We construct algorithms for verification satisfiability and truth statements in the logic $\mathbf{T}_{\mathbf{K n}}^{\mathbf{E m}, \mathbf{I n t}}$. Found computational algorithms are based at refutability of rules in reduced from at special finite frames of effectively bounded size. We show that our chosen framework is rather flexible and it allows to express various approaches to uncertainty and formalizing meaning of the embodied agent.
\end{abstract}

Key words: multi-agent logic, interacting agents, temporal logic, non-linear temporal logic, embodied agent

\section{Introduction}

This paper primarily deals with models for computational logic of multi-agent systems. In general, multi-agent systems (MAS) are collections of problemsolving entities that work together upon their environment for achieving both their individual goals and their joint goals. Development and modeling MAS may integrate many technologies and concepts from artificial intelligence (AI), CS, IT, Mathematics and other areas of computing as well as other disciplines. It is widely accepted nowadays that computational logic provides a well-defined, general, and rigorous framework for studying the syntax, semantics and procedures for the various tasks in individual agents, as well as the interaction between, and integration amongst, agents in multi-agent systems. Background of such a logic is usually multi-modal (or temporal) logic using for modeling agent knowledge modal operations $K_{i}$. In particular, this approach was usefully implemented in analysis of common and distributed agent's knowledge. A collection of summarized to 1996 research outputs may be found in e.g. Fagin et al [10]. Tools of this technique take issue in multi-agent epistemic logic. They help to describe the properties (specifications) with explicit, mathematically preciseness, which simplifies identification. 
These techniques use logical languages for reasoning about agent's knowledge and properties (e.g. various technique of mathematical (symbolic) logic is widely used (cf. $[12,13]$ ); in particular, multi-agent modal logics were implemented. Logical language is turned out to be indeed useful for these aims, cf. for a summary, - Wooldridge, 2000, [33].

Initiation of usage of logical language in knowledge representation may also be referred to e.g. Brachman and Schmolze (1985, [7]), Moses and Shoham (1993, [14]), Nebel (1990, [16]), Quantz and Schmits (1994, [21]), Rychtycki (1996, [32]).

Though technique and research outputs in MAS are various, diverse and work well in many contemporary areas, it seems, most popular area is applications in IT, - cf. Nguyen et al [18-20], Arisha et al [1], Avouris [2], Hendler [11]. Nonetheless, pure theoretical research for logic of MAS is also very popular. In particular, it was connected with attempts to clearly formalize what is a shared knowledge and what is a common knowledge It seems, first ideas concerning these problems appeared in Barwise (1988, [8]), Niegerand and Tuttle (1993, [17]), Dvorek and Moses (1990, [9]). Since a time, an approach to common knowledge logics in multi-modal framework was summarized in the book Fagin R., Halpern J., Moses Y., Vardi M. (1995, [10]).

In modeling of multi-agents reasoning an important question is how to represent interaction of agents, exchange of information (cf. e.g., Sakama et al [22]). Study of multi-modal agents logics and temporal agents-logics, representing these features, were undertaken in a series of works of the author. A kernel part in these works was representation the case when the logics describe interacting agents. In Rybakov, 2009, [28] some technique to handle interactions was found, and, as a consequence, it was proved that the multi-agent Linear Temporal Logic (with UNTIL and NEXT and with interacting agents, or dually, common knowledge) is decidable (in particular, - that the satisfiability problem for this logic is also decidable) and some algorithms solving the problem were found (cf. also Rybakov [27]). Besides, research of just multi-agent logics (as modal and temporal) with aim to find solution of satisfiability problem was earlier undertaken in Rybakov [29,30], Babenyshev and Rybakov [3-6]. Recently solution for satisfiability problem in non-linear temporal logic with only interacting agents was found in McLean and Rybakov [31].

The current paper considers non-linear temporal multi-agent logic $\mathbf{T}_{\mathbf{K n}}^{\mathbf{E m} \text {,Int }}$ with embodied agent. Here we model interaction of the agents and various aspects for computation of uncertainty in multi-agent environment. Paper suggests an algorithm for verification satisfiability and truth statements in the logic

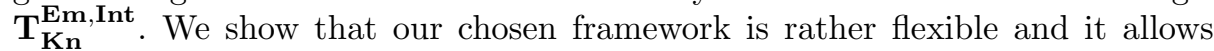
to express various approaches to uncertainty and formalizing meaning of the embodied agent.

\section{Background, Basic Notation, Definitions, Preliminaries}

The viewpoint on essence of an embodied agent might be diverse, depending on chosen model and intended implementations. Though rather common view is 
that an embodied agent is an interface agent: an intelligent agent that interacts with the environment through a physical body within that environment. We would like to model this understanding by semantics based at logical KripkeHintikka like models representing branching time including (standard but interacting) agents (it is also a point of novelty here). The second aim is to represent in this framework the conception of uncertainty via agent's interaction and embodied agent.

The basic background idea of our representation is: we have a web network with local cluster of web-network connections available for a local admin (embodied agent, interface agent), yet we have a whole network of connections, represented by web links (we interpret links forward as the time). To follow this line we start from description of a symbolic model for such representation.

The models for our semantics are based upon standard models for branching time with new subsidiary operations. In more details, Kripke/Hintikka-like frame $\mathbf{F}$ in our approach is a model $\mathbf{F}:=\left\langle W, R, R_{e}, R_{1}, \ldots, R_{n}\right\rangle$, where $W$ is a (base) set of states (worlds, which model web sites). Properties and essence of the operations in this frame are described below.

\section{Essence of the binary operations in frames}

The relation $R$ is a binary relation on $W$ (Time-relation, it models, for example, web connections, or runs of computations. Then $a R b$ means that there is a web-connection from state $a$ to state $b$ (e.g. by clicking link buttons, some amount of steps in a computational procedure, etc.)). We view at $R$ as time; it is assumed to be reflexive and transitive (which corresponds well with (i) standard understanding of time in a run of a computation, and (ii) models transitions in runs of computations, (iii) passing via web connections, etc). Formally we may fix this by laws laid upon the frame: $\forall a \in W, a R a ; \quad \forall a, b, c \in W, a R b \& b R c \Rightarrow a R c$. The states from $\mathbf{F}$ - symbols from $\mathrm{W}$ - form with respect to $R$ clusters. A cluster $C(a)$ generated by $a \in W$ is the set $\{b \mid b \in W, a R b \& b R a\}$.

The relation $R_{e}$ is a binary relation on $W$ for an embodied agent: interface relation. We assume $R_{e}$ to be the equivalence relations on any $C(a)$, where

$$
\forall b, c \in C(a)\left(b R_{e} c\right) ; \forall b, c \in C(a)\left(b R_{e} c\right) ; \text { i.e. } \forall b, c \in C(a) \forall i\left[\left(b R_{i} c\right) \Rightarrow\left(b R_{e} c\right)\right]
$$

The background for this definition is that $R_{e}$ is the relation for the embodied agent: interface relation. That is the interface agent may achieve via web links any web site in the zone of its responsibility (within $C(a)$ ). And the links within this zone are reversible, it may do any backtrack. This, it seems, corresponds very well with standard understanding of local admin in a network. Next, any relation $R_{i}$ (agent $i$ accessibility relation) is reflexive, transitive and symmetric relation (i.e. $a R b \Rightarrow b R a$ ) on $C(a)$ for any $a \in W$. It corresponds one-to one with definitions of $R_{i}$ in standard multi-agent model with autonomous agents.

It is relevant do say that the definition of an agent is the subject of much controversy in the field of Human-Computer Interaction. For example, the agent operate in the interface, as opposed to in the background or "back end" of an application. But it is an often case when the agent acts autonomously, as opposed to having a sequential conversation with the user. Often, an agent will 
satisfy one or the other of these characteristics, but it is rare that it will exhibit both at once. With this observations, the suggested above modeling of agent's knowledge is rather inexact, but yet widely accepted and reflects well many important features of knowledge representation. Later we will suggest several new ways to subtilize it in our modeling; they will be naturally defined within our approach.

The interpretation of agent's accessibility relation $R_{i},-$ via eg. internet connections, - is as follows: being logged at a web-site $a, i$-agent may access by $R_{i}$ some other web sites from the cluster $C(a)$ (in accordance with possession of access rules/passwords) - and switch between sites in its disposal freely, back and forth. Yet $i$ cannot jump to another sites outside $C(a)$ without permitting (convoy) from administrator. Say, also we may interpret relations $R_{i}$ as computational runs: there are several computational threads imitated as relations $R_{i}$ - any thread is a computational agent, while the relation $R_{e}$ holds a cluster of local computations around an time tick.

To express and elicit information which might be collected and computed via this framework we will use language with syntax based at a hybrid of a non-linear temporal logic and some multi-agent logic with new subsidiary logical operations. Language of our logic consists of standard language of Boolean logic extended with temporal and agent knowledge operations. So, it contains potentially infinite set of propositional letters $P$. Its logical operations include standard Boolean logical operations and usual unary agent knowledge operations $K_{i}, 1 \leq i \leq m$ and the unary operation $K_{e}$ - for knowledge of the embodied agent. It, as well as in [31], contains the operation for knowledge via agent's interaction KnI. This operation is a dual counterpart of the common knowledge operation introduced, e.g. in Fagin et al [10]) for common knowledge in multi-modal logic. The language also contains the unary logical operation U with meaning 'uncertain'.

Later on we will introduce some more logical operations definable in the chosen language. To express dynamics of current processes we also directly use unary temporary operations $\mathbf{P}^{+}$(with meaning 'possible in future' by a sequence of computational steps) and $\mathbf{P}^{-}$(with meaning possible, so to say in past, - by a sequence of backtracks ). The formation rules for formulas are standard: any propositional letter is a formula,

(i) if $\alpha$ and $\beta$ are formulas, then $\alpha \wedge \beta$ is a formula;

(ii) if $\alpha$ and $\beta$ are formulas, then $\alpha \vee \beta$ is a formula;

(iii) if $\alpha$ and $\beta$ are formulas, then $\alpha \rightarrow \beta$ is a formula;

(iv) if $\alpha$ is a formula, then $\mathbf{P}^{+} \alpha$ is a formula;

(v) if $\alpha$ is a formula, then $\mathbf{P}^{-} \alpha$ is a formula;

(vi) if $\alpha$ is a formula, then for any $i$ ( and for $i=e$ ) $K_{i} \alpha$ is a formula;

(vii) if $\alpha$ is a formula, then $\mathbf{K n I} \alpha$ is a formula;

(vii) if $\alpha$ is a formula, then $\mathrm{U} \alpha$ is a formula.

Accepted meaning for these operations is as follows. $K_{i} \varphi$ means: the agent $i$ knows $\varphi$ in the current state; $\mathbf{P}^{+} \varphi$ says that there is a state (web site) $b$ accessible from the current state $a$ by a sequence of links, were the statement (formula) $\varphi$ is true at $b$. So to say, there is a state, accessible in future, where $\varphi$ is true. $\mathbf{P}^{-} \varphi$ 
means that there is a state $b$ accessible from the current state $a$ by a sequence of backtracks, were the statement (formula) $\varphi$ is true at $b$.

In own turns, $\mathbf{K n I} \varphi$ means: in the current state, the statement $\varphi$ may be known by interaction between agents. $\mathrm{U} \varphi$ has meaning the statement $\varphi$ is uncertain (has uncertain truth value).

\section{Computational Rules}

Computational rules for truth of compound formulas (statements) are as follows. Assume we have given a frame $\mathbf{F}:=\left\langle W, R, R_{e}, R_{1}, \ldots, R_{n}\right\rangle$, a set of propositional letters $P$ and a valuation $V$ of $P$ in $\mathbf{F}$ which is a mapping of $P$ into the set of all subsets of the set $W$. Thus, $\forall p \in P, V(p) \subseteq W$. If, for an element $a \in W, a \in V(p)$ we say that the statement $\mathrm{p}$ is true in the state a. In the notation below $(\mathbf{F}, a) \Vdash_{V} \varphi$ is meant to say the formula $\varphi$ in true at the state $a$ in the model $\mathbf{F}$ w.r.t. the valuation $V$. The rules for computation of truth values of compound formulas are given below:

$$
\begin{aligned}
& \forall p \in P, \forall a \in W \quad(\mathbf{F}, a) \Vdash_{V} p \Longleftrightarrow a \in V(p) ; \\
& (\mathbf{F}, a) \Vdash_{V} \varphi \wedge \psi \Longleftrightarrow\left[(\mathbf{F}, a) \Vdash_{V} \varphi \text { and }\left[(\mathbf{F}, a) \Vdash_{V} \psi\right] ;\right. \\
& (\mathbf{F}, a) \Vdash_{V} \varphi \vee \psi \Longleftrightarrow\left[(\mathbf{F}, a) \Vdash_{V} \varphi \text { or }\left[(\mathbf{F}, a) \Vdash_{V} \psi\right] ;\right. \\
& (\mathbf{F}, a) \Vdash_{V} \varphi \rightarrow \psi \Longleftrightarrow\left[n o t\left[(\mathbf{F}, a) \Vdash_{V} \varphi\right] \text { or }\left[(\mathbf{F}, a) \Vdash_{V} \psi\right] ;\right. \\
& (\mathbf{F}, a) \Vdash_{V} \neg \varphi \Longleftrightarrow \operatorname{not}\left[\left(\mathbf{F}_{C}, a\right) \Vdash_{V} \varphi\right] ; \\
& \left.(\mathbf{F}, a) \Vdash_{V} K_{i} \varphi \Longleftrightarrow \text { (and for } i=e\right) \forall b \in W\left[\left(a R_{i} b\right) \Longrightarrow(\mathbf{F}, b) \vdash_{V} \varphi\right] \text {; } \\
& (\mathbf{F}, a) \Vdash_{V} \mathbf{P}^{+} \varphi \Longleftrightarrow \exists b \in W\left[(a R b) \text { and }(\mathbf{F}, b) \Vdash_{V} \varphi\right] \text {; } \\
& (\mathbf{F}, a) \Vdash_{V} \mathbf{P}^{-} \varphi \Longleftrightarrow \exists b \in W\left[(b R a) \text { and }(\mathbf{F}, b) \Vdash_{V} \varphi\right] ; \\
& (\mathbf{F}, a) \Vdash_{V} \mathbf{K n I} \varphi \Leftrightarrow \exists a_{i 1}, a_{i 2}, \ldots, a_{i k} \in W \\
& \left.\left[a R_{i 1} a_{i 1} R_{i 2} a_{i 2} \ldots R_{i k} a_{i k}\right] \&\left(\mathbf{F}, a_{i k}\right) \vdash_{V} \varphi\right] ; \\
& (\mathbf{F}, a) \Vdash_{V} \mathrm{U} \varphi \Longleftrightarrow\left[(\mathbf{F}, a) \vdash_{V} \mathbf{K n I} \varphi \quad \text { and } \quad(\mathbf{F}, a) \Vdash_{V} \mathbf{K n I} \neg \varphi\right] ;
\end{aligned}
$$

So, as in [31], we assume that a statement $\varphi$ has the uncertain truth value in the current world (state) if agents may, passing to each other information, conclude that $\varphi$ might be true in some state of the current environment, but that $\varphi$ can also be false in some state.

\section{Approach using embodied agent and other variations of uncertainty}

Another understanding of uncertainty might be given via embodied agent:

$$
(\mathbf{F}, a) \vdash_{V} \mathrm{U} \varphi \Longleftrightarrow\left[(\mathbf{F}, a) \vdash_{V} K_{e} \varphi \quad \text { and } \quad(\mathbf{F}, a) \Vdash_{V} K_{e} \neg \varphi\right] \text {. }
$$


That is we think that $\varphi$ is uncertain if the embodied agent may discover that it is somewhere true and somewhere false. It is stronger version of uncertainty comparing with the one suggested above since knowledge on the embodied agent may be bigger than the one for all agents obtained via their interaction. Yet more stronger version of uncertainty may be expressed via possibility to discover contradictory information in both future and past:

$$
(\mathbf{F}, a) \Vdash_{V} \mathrm{U} \varphi \Longleftrightarrow\left[(\mathbf{F}, a) \Vdash_{V} \mathbf{P}^{\operatorname{Sign}_{a}} \varphi \quad \text { and }(\mathbf{F}, a) \Vdash_{V} \mathbf{P}^{\operatorname{Sign}_{b}} \neg \varphi,\right]
$$

where $\operatorname{Sign}_{a}, \operatorname{Sign}_{b} \in\{+,-\}$. That is in this view, $\varphi$ is uncertain if regardless where - in future or past - this statement might be true and might be false. This is rather strongest version of uncertainty within our accepted model. A variation which is weaker is:

$$
(\mathbf{F}, a) \Vdash_{V} \mathrm{U} \varphi \Longleftrightarrow\left[(\mathbf{F}, a) \Vdash_{V} \mathbf{P}^{\operatorname{Sign}_{a}}(\mathbf{K n I} \varphi \wedge \mathbf{K n I} \neg \varphi)\right] .
$$

This is a weaker but more subtle approach - the statement $\varphi$ is uncertain if somewhere in past or future there is a state where agents via their interaction may discover that it is true and that it is false.

So, the approach we suggest is rather flexible and may express very various views on uncertainty. It is important to say, that definitions for our computation of uncertainty work similarly for all pointed approaches and we may accept any we wish for final postulating our logic.

Now on we would like to point another possible definitions for knowledge of embodied agent. We may use:

$$
\left.(\mathbf{F}, a) \Vdash_{V} K_{e} \varphi \Longleftrightarrow\left[(\mathbf{F}, a) \Vdash_{V} \mathbf{P}^{+} \varphi \vee \mathbf{P}^{-} \neg \varphi\right)\right]
$$

This is rather drastically differs from the one offered earlier, and it interprets knowledge of the embodied agent not as purely knowledge, but as to point that embodied agent definitely may always discover that $\varphi$ is true in future or otherwise in past. Again, we may accept for our approach this definition as well. Now we need to recall some definitions necessary for the sequel.

Given a model $\mathcal{M}:=\langle\mathbf{F}, V\rangle$ based at a frame $\mathbf{F}$ with a base set $W$ and a valuation $V$, and a formula $\varphi$, (i) $\varphi$ is satisfiable in $\mathcal{M}$ (denotation $-\mathcal{M} \Vdash \operatorname{Sat}_{\text {Sat }}$ ) if there is a state $b$ of $\mathcal{M}(b \in W)$ where $\varphi$ is true: $(\mathbf{F}, b) \vdash_{V} \varphi$. (ii) $\varphi$ is valid in $\mathcal{M}$ (denotation $-\mathcal{M} \Vdash \varphi)$ if, for any $b$ of $W$, the formula $\varphi$ is true at $b\left((\mathbf{F}, b) \vdash_{V} \varphi\right)$ w.r.t. $V$.

For a frame $\mathbf{F}$ and a formula $\varphi, \varphi$ is satisfiable in $\mathbf{F}$ (denotation $\mathbf{F} \vdash_{S a t} \varphi$ ) if there is a valuation $V$ in the frame $\mathbf{F}$ such that $\langle\mathbf{F}, V\rangle \Vdash{ }_{S a t} \varphi . \varphi$ is valid in $\mathbf{F}$ (notation $\mathbf{F} \Vdash \varphi)$ if $\operatorname{not}(\mathbf{F} \Vdash \operatorname{Sat} \neg \varphi)$.

Definition 1. The logic $\mathbf{T}_{\mathbf{K n}}^{\mathbf{E m}, \mathbf{I n t}}$ is the set of all formulas which are valid in all frames $\mathbf{F}$ (i.e. valid at all frames w.r.t. all valuations). A formula $\varphi$ is said to be a theorem of $\mathbf{T}_{\mathbf{K n}}^{\mathbf{E m}, \mathbf{I n t}}$ if $\varphi \in \mathbf{T}_{\mathbf{K n}}^{\mathbf{E m}, \mathbf{I n t}}$. 
We say a formula $\varphi$ is satisfiable iff there is a valuation $V$ in a Kripke frame F which makes $\varphi$ satisfiable: $\langle\mathbf{F}, V\rangle \Vdash{ }_{S a t} \varphi$. Clearly, a formula $\varphi$ is satisfiable iff $\neg \varphi$ is not a theorem of $\mathbf{T}_{\mathbf{K n}}^{\mathbf{E m}, \mathbf{I n t}}: \neg \varphi \notin \mathbf{T}_{\mathbf{K n}}^{\mathbf{E m} \text {,Int }}$, and vice versa, $\varphi$ is a theorem of $\mathbf{T}_{\mathbf{K n}}^{\mathbf{E m} \text {,Int }}\left(\varphi \in \mathbf{T}_{\mathbf{K n}}^{\mathbf{E m}, \mathbf{I n t}}\right)$ if $\neg \varphi$ is not satisfiable.

The prime aim of our paper is to find algorithm which may compute satisfiability in this logic and to compute if a statement if logically true - is a theorem. That is a very popular goal in Logic in Computer Science and AI.

\section{Computation of Satisfiability and Truth}

In this section we will use the approach borrowed from our work [31], which will be very convenient to implement for our case (actually it is just extension to implement embodied agent and new conceptions for uncertainty). The main step we need is transformation of formulas to the ones with no nested modalities at all i.e. - temporal, agents knowledge and other operations, and yet the formula in question to be just a disjunction of conjuncts with only letters, applications of modal-like operations to the letters, or yet their negations. For this, we initially convert formulas to rules and then use ready technique. The representation of formulas in such form is necessary to find algorithms (to avoid infinite loops or chains).

To recall notation and definitions, a rule $\mathbf{r}$ is an expression in the form $\mathbf{r}:=$ $\frac{\varphi_{1}\left(x_{1}, \ldots, x_{n}\right), \ldots, \varphi_{l}\left(x_{1}, \ldots, x_{n}\right)}{\psi\left(x_{1}, \ldots, x_{n}\right)}$. Here the expressions $\varphi_{1}\left(x_{1}, \ldots, x_{n}\right), \ldots, \varphi_{l}\left(x_{1}, \ldots, x_{n}\right)$ and $\psi\left(x_{1}, \ldots, x_{n}\right)$ are formulas constructed out of letters $x_{1}, \ldots, x_{n}$. The letters $x_{1}, \ldots, x_{n}$ are the variables of $\mathbf{r}$, we use the notation $x_{i} \in \operatorname{Var}(\mathbf{r})$. A meaning of a rule $\mathbf{r}$ is that the statement (formula) $\psi\left(x_{1}, \ldots, x_{n}\right)$ follows from statements (formulas) $\varphi_{1}\left(x_{1}, \ldots, x_{n}\right), \ldots, \varphi_{l}\left(x_{1}, \ldots, x_{n}\right)$. Recall definition from [31]:

Definition 2. A rule $\mathbf{r}$ is said to be valid in a Kripke model $\langle\mathbf{F}, V\rangle$ (notation $\left.\mathbf{F} \vdash_{V} r\right)$ if $\left[\forall a\left((\mathbf{F}, a) \vdash_{V} \bigwedge_{1 \leq i \leq l} \varphi_{i}\right)\right] \Rightarrow \forall a\left((\mathbf{F}, a) \vdash_{V} \psi\right)$. Otherwise we say $\mathbf{r}$ is refuted in $\mathbf{F}$, or refuted in $\mathbf{F}$ by $V$, and write $\mathbf{F} \nVdash_{V} \mathbf{r}$. A rule $\mathbf{r}$ is valid in a frame $\mathbf{F}$ (notation $\mathbf{F} \Vdash \mathbf{r}$ ) if, for any valuation $V, \mathbf{F} \vdash_{V} \mathbf{r}$

For any formula $\varphi$ we can convert it into the rule $x \rightarrow x / \varphi$. Clearly,

Lemma 1. A formula $\varphi$ is a theorem of $\mathbf{T}_{\mathbf{K n}}^{\mathbf{E m}, \mathbf{I n t}}$ iff the rule $(x \rightarrow x / \varphi)$ is valid in any frame $\mathbf{F}$.

A rule $\mathbf{r}$ is said to be in reduced normal form if $\mathbf{r}=\varepsilon / x_{1}$ where

$$
\begin{gathered}
\varepsilon:=\bigvee_{1 \leq j \leq l}\left(\bigwedge _ { 1 \leq i \leq n } \left[x_{i}^{t(j, i, 0)} \wedge\left(\mathbf{P}^{+} x_{i}\right)^{t(j, i, 1)} \wedge\left(\mathbf{P}^{-} x_{i}\right)^{t(j, i, 2)} \wedge\left(\neg \mathbf{K}_{e} \neg x_{i}\right)^{e_{j, i}} \wedge\right.\right. \\
\left.\left.\bigwedge_{1 \leq q \leq n}\left(\neg \mathbf{K}_{q} \neg x_{i}\right)^{t(j, i, q, 1)} \wedge \mathbf{K n I} x_{i}^{t(j, i, 3)} \wedge\left(\mathrm{U} x_{i}\right)^{t(j, i, 4)}\right]\right),
\end{gathered}
$$

all $x_{s}$ are certain letters (variables), $t(j, i, z), t(j, i, k, z), e_{j, i} \in\{0,1\}$ and, for any formula $\alpha$ above, $\alpha^{0}:=\alpha, \alpha^{1}:=\neg \alpha$. 
Definition 3. Given a rule $\mathbf{r}_{\mathbf{n f}}$ in reduced normal form, $\mathbf{r}_{\mathbf{n f}}$ is said to be a normal reduced form for a rule $\mathbf{r}$ iff, for any frame $\mathbf{F}, \mathbf{F} \Vdash \mathbf{r} \Leftrightarrow \mathbf{F} \Vdash \mathbf{r}_{\mathbf{n f}}$.

Theorem 1. There exists an algorithm running in (single) exponential time, which, for any given rule $\mathbf{r}$, constructs its normal reduced form $\mathbf{r}_{\mathbf{n f}}$.

For readers interested in proof of this statement, cf. Theorem 1 and its proof in [31]. As we know, the decidability of our logic (in particular decidability of the satisfiability problem) will follow (by this theorem) if we find an algorithm recognizing rules in reduced normal form which are valid in all frames $\mathbf{F}$. Very important starting point to implement this technique is to efficiently bound the size of clusters under consideration in order to efficiently define the interaction of agents. As in [31] we will use the same step as been earlier implemented in Lemma 8 in Rybakov [28] for simply linear temporal multi-agent logic.

Lemma 2. A rule $\mathbf{r}_{\mathbf{n f}}$ in reduced normal form is refuted in a frame $\mathbf{F}$ if and only if $\mathbf{r}_{\mathbf{n f}}$ can be refuted in a frame with time clusters of size square exponential from $\mathbf{r}_{\mathbf{n f}}$.

If this is curried out, the rest is a standard work using filtration technique and other instruments of non-classical mathematical logic. As result we obtain

Lemma 3. A rule $\mathbf{r}_{\mathbf{n f}}$ in reduced normal form is refuted in a frame $\mathbf{F}$ iff $\mathbf{r}_{\mathbf{n f}}$ can be refuted in a finite frame $\mathbf{F}_{1}$ by a valuation $V$, where the size of the frame $\mathbf{F}_{1}$ has effective upper bound computable from the size of $\mathbf{r}_{\mathbf{n f}}$.

Based at Theorem 1, Lemma 1 and Lemma 3 we obtain our main technical result: an algorithm for computation of satisfiability and decidability of our logic.

Theorem 2. The logic $\mathbf{T}_{\mathbf{K n}}^{\mathbf{E n}, \mathbf{I n t}}$ is decidable; the satisfiability problem for $\mathbf{T}_{\mathbf{K} \mathbf{n}}^{\mathbf{E m}, \mathbf{I n t}}$ is decidable.

\section{Conclusion, Future Work}

We investigate non-linear temporal multi-agent logic $\mathbf{T}_{\mathbf{K n}}^{\mathbf{E m}, \mathbf{I n t}}$ with embedded agent. In suggested framework we model interaction of the agents and various aspects for definition of uncertainty in multi-agent environment. The aim of the paper is to construct algorithms for verification satisfiability and truth statements for $\mathbf{T}_{\mathbf{K n}}^{\mathrm{Em}, \mathbf{I n t}}$. We find computational algorithms based at refutability of rules in reduced from at special finite frames of effectively bounded size. It is shown that our chosen framework is rather flexible and allows to handle various approaches to uncertainty and definitions of the embedded agent.

Future subsequent research may concern various aspects in suggested approach. In particular, pointed technique may be extended to handle more subtle aspects of agents interaction and duties of the embodied agent. E.g. interaction of agents is represented now as just passing information, without considering intermediate conflicts, voting etc. Functions of the embodied agent are also shown 
as pure universal modality or modal-like operation of kind possible. Pure logical problems, as axiomatizability, complexity issues are open ap to now. Yet it is interesting to extend our approach to components of fuzzy logic - with numeric values for agents knowledge and believes.

\section{References}

1. Arisha K., Ozcan F., Ross R., Subrahmanian V.S., Eiter T. and Kraus S. Impact: A platform for collaborating agents, IEEE Intelligent Systems 14, 1999 (2), pp. 64-72.

2. Avouris N.M. Co-operation knowledge-based systems for environmental decisionsupport, Knowledge-Based Systems 8 (1995), (1), pp. 39-53.

3. Babenyshev S., Rybakov V. Logic of Plausibility for Discovery in Multi-agent Environment Deciding Algorithms. KES (3) 2008: Lecture Notes in Computer Science, 2008, Volume 5179/2008, pp. 210-217.

4. Babenyshev S., Rybakov V. Decidability of Hybrid Logic with Local Common Knowledge Based on Linear Temporal Logic LTL. CiE 2008: Lecture Notes in Computer Science, 2008, Volume 5028/2008, pp. 32-41.

5. Babenyshev S., Rybakov V. Logic of Discovery and Knowledge: Decision Algorithm. KES (2), Lecture Notes in Computer Science, 2008, Volume 5178/2008, pp. 711-718

6. Babenyshev S., Rybakov V. Describing Evolutions of Multi-Agent Systems. KES (1) 2009: Lecture Notes in Computer Science, 2009, Volume 5711/2009, pp. 38-45.

7. Brachman R.J., Schmolze J. G., An overview on the KL-ONE knowledge representation system. - Cognitive Science, 9(2), 1985, 179 -226.

8. Barwise J. Three Views of Common Knowledge. - In Vardi (Ed.). Proc. Second Confeuence on Theoretical Aspects of Reasoning about Knowledge (1988), San Francisco. Califounia, Morgan Kaufmann, 365 - 379.

9. Dwork C., and Moses Y. Knowledge and Common Knowledge in a Byzantine Environment: Crash Failures . - Information and Computation, Vol. 68 (1990), No. 2, 156 - 183.

10. Fagin R., Halpern J., Moses Y., Vardi M. Reasoning About Knowledge - Book, The MNT Press, Cambridge, Massachusetts, London, England, 1995, 410 pp.

11. Hendler J. Agents and the semantic web, IEEE Intelligent Systems, 16 (2001) (2), pp. 30-37.

12. Kifer M., Lozinski L. A Logic for Reasoning with Inconsistency. - J. Automated Deduction, Vol 9 (1992), 171 - 115.

13. Kraus S., Lehmann D.L. Knowledge, Belief, and Time.- Theoretical Computer Science, Vol. 98 (1988), 143 - 174.

14. Moses Y., Shoham Y. Belief and Defeasible Knowledge. - Artificial Intelligence, Vol. 64 (1993), No. 2, 609 - 322.

15. D. McLean and V. Rybakov. Multi-Agent Temporary Logic TS $4_{K_{n}}^{U}$ Based at Nonlinear Time and Imitating Uncertainty via Agents Interaction. - Artificial Intelligence and Soft Computing, 2013, Springer, Berlin-Heidelberg, 2013, 375-384.

16. Nebel B. Reasoning and Revision in Hybrid Representation Systems. - Lecture Notes in Computer Science, vol. 322 (1940) Springer Cerlag.

17. Neiger G., Tuttle M.R. Common knowledge and consistent simultaneous coordination. - Distributed Computing, Vol 5 (1993), No. 3, 334 - 352. 
18. Nguyen N.T. et al. (2008, Eds): Agent and Multi-agent Systems: Technologies and Applications. Proceedings of KES-AMSTA 2008. Lecture Notes in Artificial Intelligence 4953. Springer-Verlag.

19. Nguyen N.T., Huang D.S. (2009): Knowledge Management for Autonomous Systems and Computational Intelligence. Journal of Universal Computer Science $15(4)$.

20. Nguyen N.T., Katarzyniak R. (2009): Actions and Social Interactions in Multiagent Systems. Special issue for International Journal of Knowledge and Information Systems 18(2).

21. Quantz J., Schmitz B., Knowledge-based disambiguation of machine translation. Minds and Machines (9996), 9: 99 - 97. ccc

22. Sakama Ch. and Cao Son T. Interactind Answer Set, Computational Logic in Multi-Agent Systems: 10th International Congress, LNAI, Springer, Vol. 6214, 2010, pp. $122-140$.

23. Rybakov V.V. A Criterion for Admissibility of Rules in the Modal System $S 4$ and the Intuitionistic Logic. - Algebra and Logic, V.23 (1984), No 5, pp. 369-384 (Engl. Translation).

24. Rybakov V.V. Admissible Logical Inference Rules. - Book: Series: Studies in Logic and the Foundations of Mathematics, Vol. 136, Elsevier Sci. Publ., North-Holland, ISBN: 0444895051, 1997.

25. Rybakov V.V. Logical Consecutions in Discrete Linear Temporal Logic. Journal of Symbolic Logic (ASL, USA), V.70, No 4 (2005), pp. 1137-1149.

26. Rybakov V. Sine-Until Temporal Logic Based on Parallel Time with Common Past. Logical Foundation of Computer Science, Lecture Notes in Computer Science, Springer, Vol. 4514, New York, USA, 2007, 486-497.

27. Rybakov V. Logic of knowledge and discovery via interacting agents - Decision algorithm for true and satisfiable statements. Inf. Sci. (Elsevier, North-Hollnd New York) 179(11): 1608-1614 (2009).

28. Rybakov V. Linear Temporal Logic $L T K_{K}$ extended by Multi-Agent Logic $K_{n}$ with Interacting Agents. - J. Log. Comput., Oxford Press, 19(6): pp. 989-1017 (2009).

29. Rybakov V. Representation of Knowledge and Uncertainty in Temporal Logic LTL with Since on Frames Z of Integer Numbers. KES (1) 2011: Lecture Notes in Computer Science, 2011, Volume 6881/2011, pp. 306-315.

30. Rybakov V. Agents' Logics with Common Knowledge and Uncertainty: Unification Problem, Algorithm for Construction Solutions. KES (1) 2011: Lecture Notes in Computer Science, 2011, Volume 6881/2011, pp. 171-179.

31. Rybakov V. Multi-Agent Logic based on Temporary Logic $T S 4_{K_{n}}$ serving Web Search. In: KES 2012, Frontiers in Artificial Intelligence and Applications, Vol. 243, Eds. Manuel Grana at al. Advances in Knowledge-Based and Intelligent Information and Engineering Systems, 2012, pp. 108 - 117.

32. Rychtyckyi N. DLMS: An evaluation of KL-ONE in the automobile industry. In Aiello L. C., Doyle J., Shapiro S., editors, Proc. of he 5-th Int. Conf. on Principles of Knowledge Representation and Reasoning (KR-96), 1996, pp. 588 - 596. Cambridge, Mass. Morgan Kaufmann, San Francisco.

33. Wooldridge M. Reasoning about rational agents. - MIT Press, 2000. 